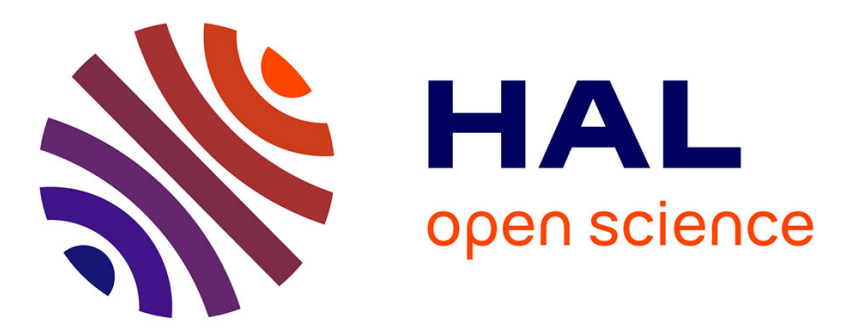

\title{
A Statistical Method for the Prediction of the Loop Tack and the Peel of PSAs from Probe test Measurements
}

Isabelle Rivals, Léon Personnaz, Costantino Creton, François Simal, Patrice

Roose, Steven van Es

\section{- To cite this version:}

Isabelle Rivals, Léon Personnaz, Costantino Creton, François Simal, Patrice Roose, et al.. A Statistical Method for the Prediction of the Loop Tack and the Peel of PSAs from Probe test Measurements. Measurement Science and Technology, 2005, 16, pp.2020-2029. 10.1088/0957-0233-16-10-018 . hal00176886

\section{HAL Id: hal-00176886 \\ https://hal.science/hal-00176886}

Submitted on 19 Mar 2013

HAL is a multi-disciplinary open access archive for the deposit and dissemination of scientific research documents, whether they are published or not. The documents may come from teaching and research institutions in France or abroad, or from public or private research centers.
L'archive ouverte pluridisciplinaire $\mathbf{H A L}$, est destinée au dépôt et à la diffusion de documents scientifiques de niveau recherche, publiés ou non, émanant des établissements d'enseignement et de recherche français ou étrangers, des laboratoires publics ou privés. 


\title{
A STATISTICAL METHOD FOR THE PREDICTION OF THE LOOP TACK AND THE PEEL OF PSAS FROM PROBE TEST MEASUREMENTS
}

\section{Isabelle Rivals', Léon Personnaz', Costantino Creton², François Simal'3, Patrice Roose $^{3}$ and Steven van $\mathbf{E s}^{3}$}

\author{
${ }^{1}$ Équipe de Statistique Appliquée, ESPCI, Paris, France. \\ ${ }^{2}$ Laboratoire de Physico-Chimie des Polymères et des Milieux Dispersés, ESPCI, Paris, \\ France. \\ ${ }^{3}$ R\&D Surface Specialties, Drogenbos, Belgium.
}

\begin{abstract}
We investigated the potential of the probe test as a high throughput test for the rapid screening of a large number of candidate pressure-sensitive-adhesives. The output of a successful screening tool should be usable to predict with some precision important characteristics of adhesives such as loop tack and peel which take longer to measure. The output of an instrumented probe tack test being both reproducible and sensitive to changes in polymer structure or formulation, we developed a statistical procedure which builds a polynomial model of the force values at the relevant time instants only, the number of necessary monomials being established using a test for lack of fit. The performance of the models is then estimated using cross-validation and an independent test set. The prediction results obtained on a data set representative of commercial adhesives show that the force signal recorded during a probe test indeed contains exploitable information about the loop tack and peel forces, and that the proposed statistical procedure is more successful for quantitative predictions than existing alternative approaches.
\end{abstract}




\section{Introduction}

Pressure-sensitive-adhesives (PSAs) are increasingly popular for fastening applications due to their safe and easy handling. With the increase in the number and variety of applications come more demanding requirements in terms of properties. In order to speed up the optimization of the properties needed for a specific application, it will be become more and more important to develop a good high throughput test for the rapid screening of a large number of materials, so as to select only the most promising ones [Grunlan et al. 2004] [Kulikov et al. 2005] [Zhang et al. 2005].

Initially, the instrumented probe tack test has been developed to gain more physical insight in the mechanisms of debonding of PSAs [Zosel 1985] [Lakrout et al. 1999] [Shull \& Creton 2004]. A careful analysis of the results obtained with the probe tack test has led to significant advances in the understanding of the micromechanisms of debonding of a PSA layer from a rigid substrate, and the main results can be found in a comprehensive review [Shull \& Creton 2004]. Most of these advances came from the very detailed information obtained from the probe test, which provides an entire stress-strain curve and not a unique value, coupled with an in situ observation of the deformation mechanisms of the adhesive layer with a video camera [Creton et al. 2001] [Brown et al. 2002] [Creton 2003].

These results have shown that for a very confined PSA layer which is being deformed in traction, failure is initiated by the formation of cavities at the interface with the probe. These cavities then form a foamed structure, which can be easily deformed to large strains in the tensile direction. Final detachment of the adhesive from the probe occurs when the nonlinear elastic properties of the adhesive display a significant strain hardening due to the finite extensibility of the polymer chains forming the backbone of the adhesive.

Despite its obvious advantages, such as speed of execution and reproducibility [Chuang et al. 1997], the probe test is not yet widely accepted in industry, where adhesive properties are still typically tested with standardized industry tests closer to applications, such as loop tack, peel or shear tests [PSTC 2000]. It would therefore be economically advantageous to be able to predict the result of a loop tack, peel or shear test, from a simple probe test experiment which typically lasts less than a minute. However, the important conceptual advances that the more fundamental investigations brought, did not yet result in a quantitative correlation between the outcome of the probe test (i.e. a curve of stress as a function of strain) and a value such as loop tack, peel force or shear, and further work is needed in that direction.

In this paper, we explore a different but parallel approach to predict the property of interest, loop tack or peel value, from a probe test curve. This approach is based on the assumption that the information needed to predict the property is contained in the curve obtained with the probe tack test, but needs to be extracted with statistical tools. From the experimental point of view, the requirements are simple: the probe test must be sensitive to changes in polymer structure and 
formulation that will have an effect on the application property, and the probe test must be reproducible to provide reliable data. The sensitivity of the test to changes in chemical structure and in formulation has been shown in several publications on the subject [Zosel 1985] [Brown et al. 2002] [Lakrout et al. 2001], and is summarized in Figure 1 which shows a typical probe test curve for a PSA. The compression and initial part of the tensile curve are sensitive to the linear viscoelastic properties of the adhesive; the peak in tension and the plateau following the peak are much more sensitive to both adhesive properties and non-linear elastic properties. Unlike a simple rheology experiment in the linear viscoelastic regime, the probe test deforms the adhesive layer, in both the linear (compression) and the non-linear (tension) regime which will be essential to predict debonding energies [Roos \& Creton 2004]. Reproducibility is usually better than for standard PSA tests, but can be further optimized by a careful sample preparation and by the choice of a hemispherical probe, which is insensitive to small misalignments [Chuang et al. 1997] [Crosby \& Shull 1997].

Therefore a suitable statistical method should be able to extract the most relevant information from the probe test curves and use them to build a predictive model of a specific property such as loop tack or peel value. It is this path that we explore in our paper. The methodology we use has been developed to extract the most significant part of a data set to build a predictive model of the desired property, see [Rivals \& Personnaz 2003a,b].

\section{Experimental}

Probe tests were performed for 26 different materials representative of commercial permanent PSAs based on SIS (Kraton D-1160 and D-1161, from Kraton Polymers) and tackifing resins (Piccotac 95E, Piccotac 212 and Foral 85-E, from Eastman Chemical Company), see Appendix 1. Three to five repeat tests were made for each material in the same conditions. The probe tests were performed on a TA-XT2 i HR texture analyzer (Stable Microsystems) fitted with a spherical probe having a diameter of $25 \mathrm{~mm}$. The probe was brought in contact with the adhesive layer at a velocity of $10 \mu \mathrm{m} / \mathrm{s}$, until a compressive force of $1.1 \mathrm{~N}$ was reached. It was kept in contact for 0.5 second at this load, and next removed at a debonding speed of $10 \mu \mathrm{m} / \mathrm{s}$. The whole force-distance curve (compression and traction) was recorded.

The adhesive films were prepared by transfer coating: a toluene solution of polymer and tackifier was applied on Silicone paper using an automatic bar coater. The drying conditions were as follows: 30 minutes at room temperature, followed by 3 minutes at $110^{\circ} \mathrm{C}$. The dried PSA film (thickness $30 \mu \mathrm{m}$ ) was then coated with a PET film (Mylar, $23 \mu \mathrm{m}$ thick). The laminate was conditioned for 24 hours under controlled humidity and temperature $\left(23^{\circ} \mathrm{C} \pm 2{ }^{\circ} \mathrm{C}\right.$, $50 \% \mathrm{RH} \pm 5 \% \mathrm{RH})$ before testing. 


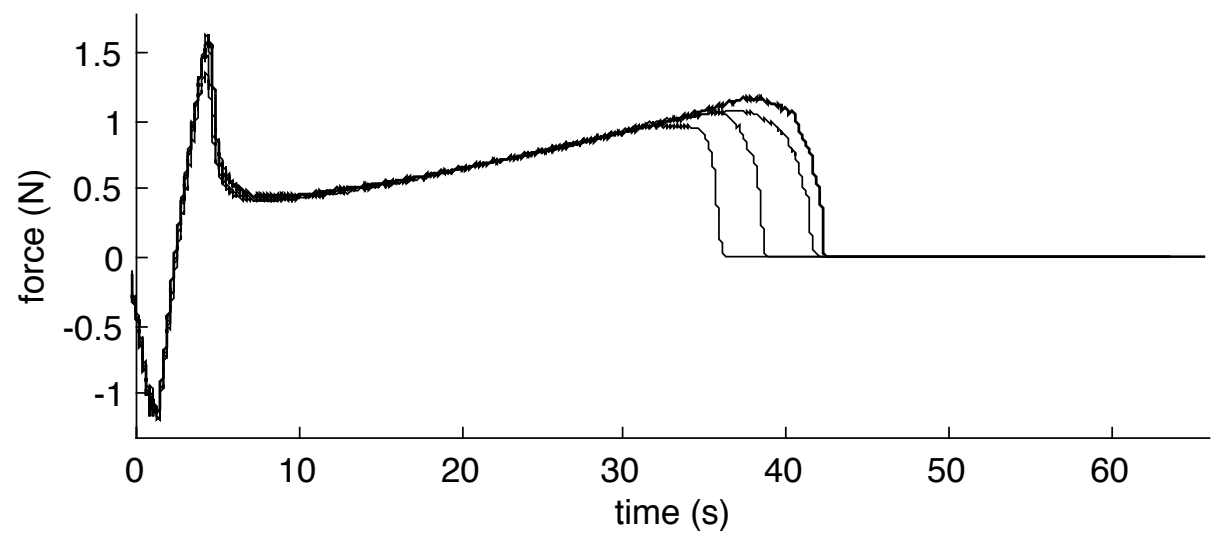

Figure 1. Typical force curves obtained with four probe test repeats (curves $\mathrm{N}^{\circ} 300$ ). The loop tack test provides a mean value of $21.2 \mathrm{~N} / 25 \mathrm{~mm}$, and the peel test a mean value of $15.5 \mathrm{~N} / 25 \mathrm{~mm}$.

The peel tests were performed using FINAT FTM 1 conditions: $25 \mathrm{~mm}$ x $90 \mathrm{~mm}$ strips of PSA were applied on stainless steel plates using a $1 \mathrm{~kg}$ FINAT standard test roller, and $180^{\circ}$ peel were measured at a peel rate of $300 \mathrm{~mm} / \mathrm{min}$, after 20 minutes. The loop tack tests were performed using FINAT FTM 9 conditions: $25 \mathrm{~mm}$ x $210 \mathrm{~mm}$ strips of PSA were applied on stainless steel plates. The loop was brought in contact and immediately removed at 300 $\mathrm{mm} / \mathrm{min}$. No stick-slip phenomenon was observed during the peel and loop tack tests.

The outcome of the experimental part of the study is a series of force curves with the corresponding values of the peel force and of the loop tack. Typical force curves obtained with 4 probe test repeats are shown on Figure 1, with the mean values of the loop tack and of the peel. Note that each repeat was performed on a fresh sample. The whole data set is described in Appendix 2.

\section{Design of the predictive models}

This section is devoted to the design of predictive models of the loop tack and of the peel from force descriptors, using the previous data set. Briefly, there are five steps: 1) the study of the variability of the loop tack and peel measurements ; 2) the choice of the candidate descriptors, i.e. of the variables that potentially contain the information about the loop tack and the peel ; 3 ) the construction of nested models of increasing complexity involving the candidate descriptors and/ or fixed functions of them ; 4) the selection of the model of minimal necessary complexity given the variability of the loop tack and peel values, i.e. involving only the most relevant descriptors ; 5) the estimation of the performance of the model using both crossvalidation and an independent test set.

\section{III.1. Variability of the loop tack and of the peel measurements}

The data set involves 26 adhesives, however, there are 2 missing values for peel (curves 277 
and 279, see Table 5 in Appendix 2). Thus, the data set size is $N=26$ for loop tack, and only $N=24$ for peel. For the 16 first adhesives, the loop tack and the peel values $\left\{y^{k}\right\}$ are the mean of $M^{k}=3$ repeat measurements $\left\{y^{k, j}\right\}$, but only the means $\left\{y^{k}\right\}$ are available (see Appendix 2, Table 5). For the last 10 adhesives (shaded cells in Table 5), the repeat measurements $\left\{y^{k, j}\right\}$ are available, and the loop tack and the peel values are the mean of $M^{k}=4$ or 5 measurements. For adhesive $k$, with $k=17$ to 26 , the estimate $\left(s^{k}\right)^{2}$ of the measurement variance $\left(\sigma^{k}\right)^{2}$ is given by:

$$
\left(s^{k}\right)^{2}=\frac{\sum_{j=1}^{M^{k}}\left(y^{k, j}-y^{k}\right)^{2}}{M^{k}-1}
$$

The values of the $\left\{s^{k}\right\}$ are shown in Figure 2 .

a)

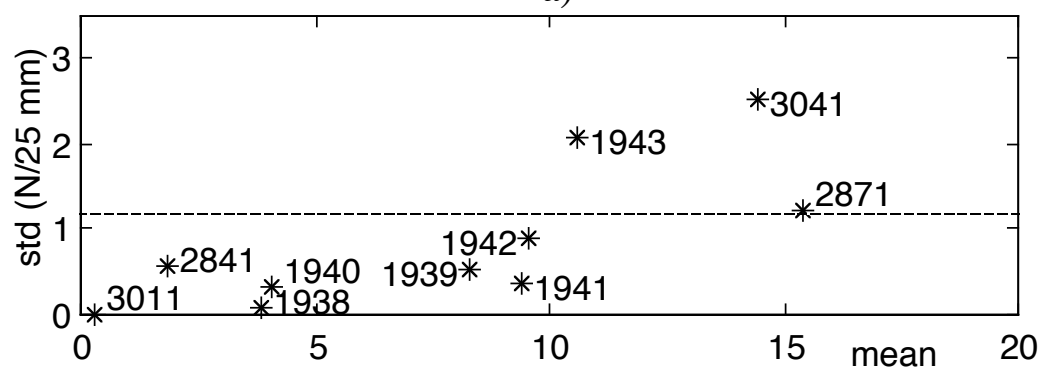

b)

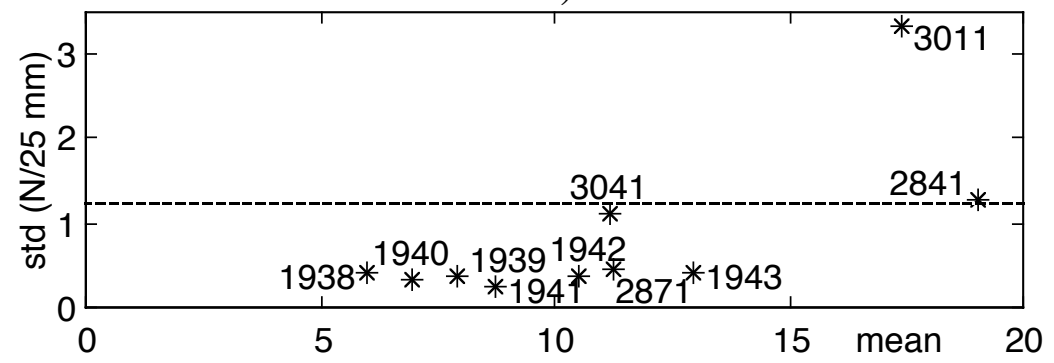

Figure 2. Local estimates $\left\{s^{k}\right\}$ of the standard deviations of the measurements obtained with repeats for the last 10 adhesives of Table 5, and mean estimate $s$ (dashed line): a) loop tack, b) peel.

The measurement noise is clearly heteroscedastic, i.e. its variance depends on the adhesive. In principle, this should lead to a weighted least squares approach. However, since the $\left\{y^{k, j}\right\}$ and hence the $\left\{s^{k}\right\}$ are available for only 10 adhesives out of 26 , such an approach is not feasible in practice, and the model parameters will be estimated using ordinary least squares, see Appendix 3.

In the following, we will use the average estimate of the standard deviation of the output. As a matter of fact, if the variances are all assumed equal to a common value $\sigma^{2}$, an estimate $s^{2}$ of $\sigma^{2}$ is computed on the available repeat measurements:

$$
s^{2}=\frac{\sum_{k=17}^{26}\left(M^{k}-1\right)\left(s^{k}\right)^{2}}{M-10} \text { with } M=\sum_{k=17}^{26} M^{k}
$$


Numerically, we obtain $s=1.19 \mathrm{~N} / 25 \mathrm{~mm}$ for loop tack and $s=1.23 \mathrm{~N} / 25 \mathrm{~mm}$ for peel, see Figure 2. These values will be used later for the selection of the appropriate model complexity with a lack of fit test, see section III.4 and Appendix 3.

\section{III.2. Choice of the candidate descriptors}

As stated in the introduction, it is assumed that the information about the loop tack and the peel properties is contained in the force-displacement curves measured during a probe test. Since the displacement is the same for each test and hence for each adhesive, only the force as a function of time is useful for the prediction of the loop tack and of the peel values.

a)
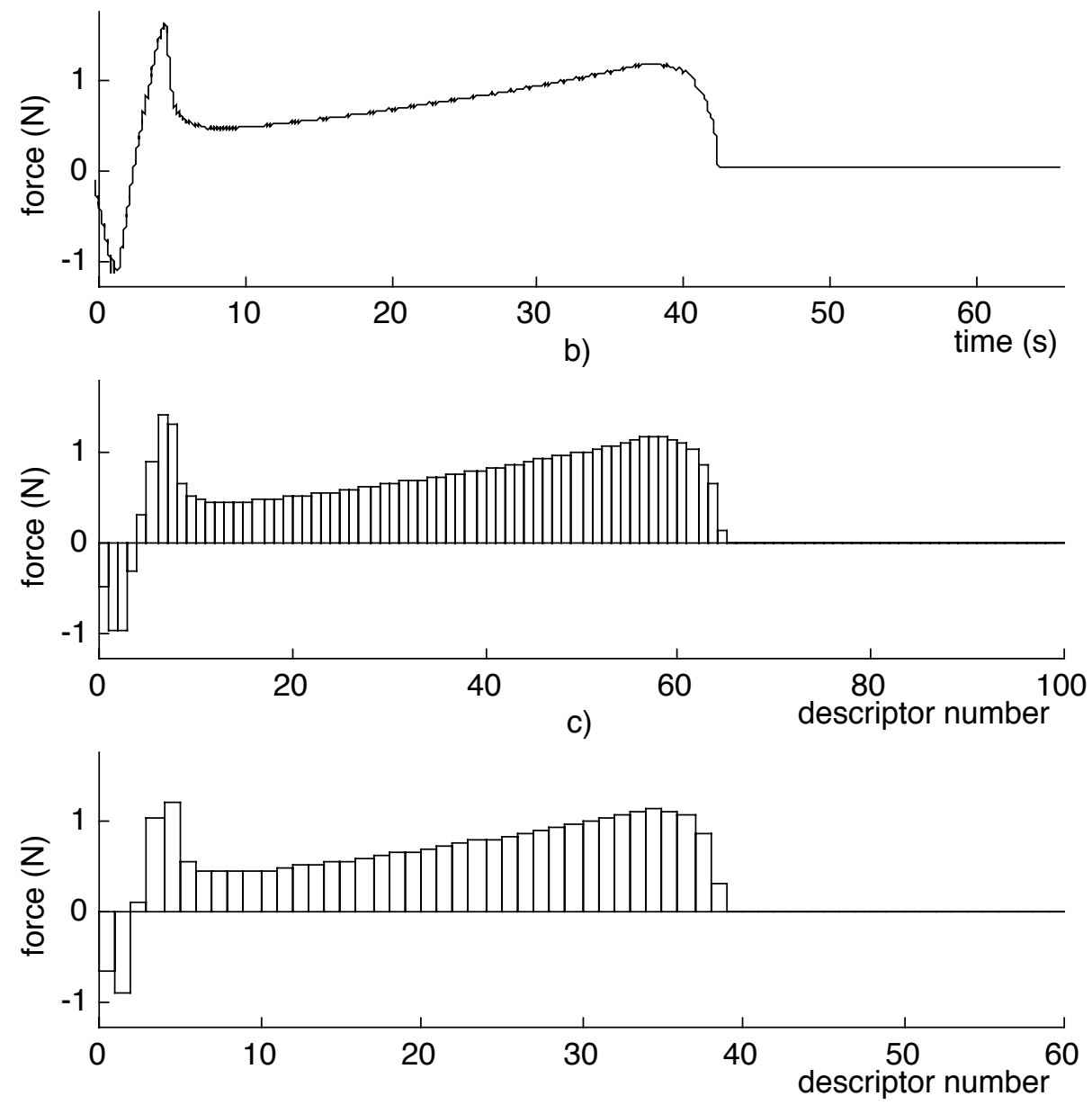

Figure 3. Thick curve of Figure $1\left(\mathrm{~N}^{\circ} 300\right)$ : a) force measurements performed every $\left.2 \mathrm{~ms} ; \mathrm{b}\right) 100$ candidate descriptors; c) 60 candidate descriptors.

In the following, the dimension of the descriptor space is the number of time instants at which the force values are considered, between the contact creation and the time corresponding to the longest test of the dataset. The force measurements are performed every 2 milliseconds, see Figure $3 \mathrm{a}$ which shows one of the curves obtained for the adhesive $\mathrm{N}^{\circ} 300$, the thick curve of Figure 1 . We performed a dimension reduction by drastically reducing the time resolution. 
Hundred descriptors, i.e. force values, seem enough to grasp the information contained in the force curve, see Figure $3 b$ (each descriptor value is the mean of the 330 corresponding force values). We even consider lower resolutions, see Figure 3c. There is a compromise between losing information and reducing the number of candidate descriptors: as shown in section IV, a good compromise is achieved for a number of descriptors equal to 60.

As stated above, the reproducibility of the probe test is quite high. However, the curves of some adhesives show a significant variability, especially at the end of the curve see Figure 1. Therefore, we choose to consider the mean of the previous descriptor values over the repeats of the probe test ( $M^{k}=3$ to 5 repeats).

An alternative choice of descriptors would be that of physically significant characteristics of the curves, such as the force minima and maxima and the corresponding time instants, the area of the curve below and above zero, etc. (see Appendix 4). We also experimented such a choice, but obtained worse results than with the raw force descriptors.

\section{III.3. Construction of nested models involving the most relevant descriptors}

We are now in the presence of the descriptors judged potentially relevant for the prediction of the loop tack or of the peel. We denote the candidate descriptors values by the $\left\{\xi_{i}\right\}_{i=1}$ to $n$, the components of the n-descriptor vector $\xi$, and by $y$ the mean value of the loop tack or of the peel. The assumption that the information about the property is contained in the candidate descriptors is equivalent to assuming the existence of a model of the form:

$$
y=g(\xi)+w
$$

where $g$ is an unknown function, and $w$ is a zero mean random variable modeling the output noise studied. The goal is hence to choose a suitable parameterized function $f(\xi, \boldsymbol{\theta})$ and a value $\boldsymbol{\theta}_{\text {opt }}$ of the parameters $\boldsymbol{\theta}$ such that $f\left(\boldsymbol{\xi}, \boldsymbol{\theta}_{\text {opt }}\right)$ is close to $g(\boldsymbol{\xi})$ in the domain of interest.

A first issue is that, the dimension of $\xi$ being large $(n \in[50 ; 100])$, even a simple affine model involves too many parameters $(n+1)$ given the number of measurements $(N=26$ for tack and 24 for peel). Thus, the design of the model heavily relies on the selection of the relevant and non redundant descriptors among the candidates. Therefore, the model must be mathematically suitable for the selection of the most relevant descriptors.

Next, the model should also be suitable for the modeling of interactions between different parts of the curve and of possible nonlinearities.

Polynomial models satisfy both conditions: they are linear in their parameters, but nonlinear in the descriptors. A polynomial of degree two is able to represent linear (monomials $\xi_{i}$ ) and quadratic (monomials $\left(\xi_{i}\right)^{2}$ ) dependencies, as well as interactions between different regions of the curve (cross-products monomials $\xi_{i} \xi_{j}$ ). The value of the degree is often limited to 2 by the available computer memory ${ }^{1}$.

\footnotetext{
1 As a matter of fact, the polynomial of degree $d$ possesses a number of monomials equal to :
} 
A third issue is how to construct polynomials involving an increasing number of the most relevant monomials: this is achieved using the modified Gram-Schmidt orthogonalization procedure [Golub 1996], the question of when to stop this construction being answered in the next subsection. Let us consider a polynomial of fixed degree $d$ of the $n$ candidate descriptors $\left\{\xi_{i}\right\}$. For example, if the list of the candidate descriptors is $\xi_{1}, \xi_{2}, \ldots, \xi_{n}$, the polynomial of degree 2 involves a constant term and the monomials: $\xi_{1}, \xi_{2}, \ldots, \xi_{n}, \xi_{1} \xi_{2}, \xi_{1} \xi_{3}, \ldots, \xi_{n-1} \xi_{n}$, $\xi_{1}^{2}, \ldots, \xi_{n}{ }^{2}$. We denote the $N$-vector of the mean loop tack or peel values by $\boldsymbol{y}$, and the $N$ vectors corresponding to the monomials by the $\left\{\boldsymbol{x}_{i}\right\}$.

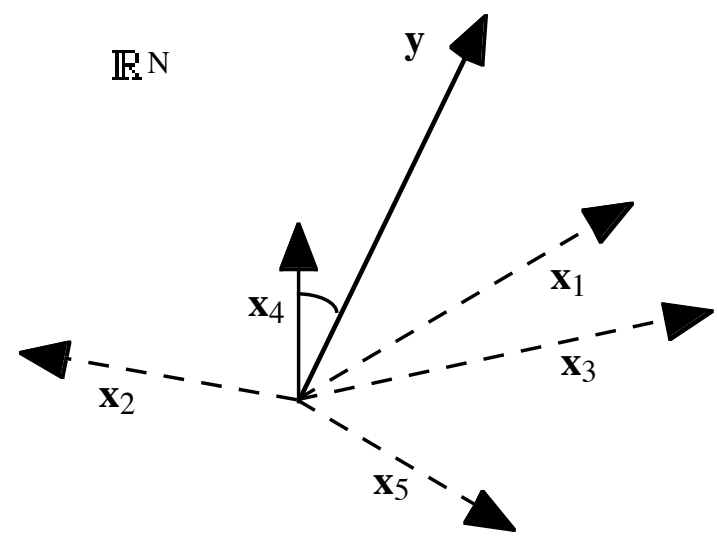

Figure 4. The vector $\left\{\mathbf{x}_{4}\right\}$ is such that $\left|\cos \left(\mathbf{y}, \mathbf{x}_{\mathbf{j}}\right)\right|$ is the largest, and is introduced first.

The monomials $\left\{\boldsymbol{x}_{i}\right\}$ are introduced according to their decreasing contribution to the explanation of $\boldsymbol{y}$. The monomial $\mathrm{j}$ that is considered to have the maximum contribution to the output is the monomial such that $\left|\cos \left(\boldsymbol{y}, \boldsymbol{x}_{j}\right)\right|$ is the largest; it is also the monomial which decreases most the residual sum of squares, see Appendix 3. The remaining monomials and the output are orthogonalized with respect to this first monomial $j$ using the Gram-Schmidt orthogonalization algorithm. The procedure is repeated in the subspace orthogonal to the first ranked monomial $j$, and so on.

An alternative to this model construction could be a principal component analysis (PCA) followed by the construction of least squares models having the first principal components as inputs. Such an approach has two main disadvantages. First, PCA is an unsupervised method: the principal components are computed from the descriptor values only, without using the output values of the propery to predict. Thus, the first principal components have no reason to

$$
N_{\text {mono }}(n, d)=\sum_{i=1}^{d} K_{n}^{i}=\sum_{i=1}^{d} C_{n+i-1}^{i}
$$

where $K_{n}^{i}$ is the number of $i$ to $i$ combinations of $n$ objects with repetitions, and $C_{n}^{i}=i ! / n !(n-i) !$ is the number of $i$ to $i$ combinations of $n$ objects without repeats. For example, the polynomial of degree $d=2$ of $n=100$ input variables possesses 5150 monomials, that of degree $d=3$ possesses 176850 monomials. 
explain the property, whereas, in our procedure, the monomials are introduced precisely in the order of their decreasing correlation with the output property. Next, PCA is a linear method: the principal components are linear combinations of the descriptors. Thus, PCA is not able to capture the influence of nonlinearities or of interactions between descriptors, whereas higherorder monomials do.

In the next subsection, we study when to stop the Gram-Schmidt model construction.

\section{III.4. Selection of the model with minimal necessary complexity}

The model with minimal necessary complexity is the model with the smallest number of monomials which achieves a precision, i.e. a mean square error, that is comparable to the variance $\lambda^{2}$ of the output. Each time a monomial is introduced, we can test whether the mean square error of the corresponding polynomial is not too large, using a lack of fit test, whose principle is detailed in Appendix 3. The first polynomial which satisfies the test is selected. The test is performed with a type I risk $\alpha=1 \%$. Since there are $M^{k}=3$ to 5 repeats for each adhesive, we make the approximation that $M^{k}=m=4$, i.e. that $\lambda^{2}=\sigma^{2} / m$. This corresponds to an estimate of $\lambda$ equal to $v=s / 2$, i.e. $v=0.59$ for loop tack and $v=0.61$ for peel.

In order to quantify the significance of the force descriptors involved in the selected model, we define the sensitivity of its output with respect to each force descriptor as:

$$
\delta_{j}=\frac{1}{N} \sum_{k=1}^{N}\left|\frac{\partial f\left(\boldsymbol{\xi}, \boldsymbol{\theta}_{L S}\right)}{\partial \xi_{j}}\right|_{k}\left|=\frac{1}{N} \sum_{k=1}^{N}\right| \sum_{i=1}^{p} \theta_{L S_{i}} \frac{\partial x_{i}}{\partial \xi_{j} \mid k}|| \text { for } j=1 \text { to } n
$$

where $N$ denotes the number of PSAs, $f\left(\xi, \boldsymbol{\theta}_{L S}\right)$ is the model output, $n$ is the number of descriptors, $p$ is the number of selected monomials, $x_{i}$ is the $i$-th selected monomial, and $\theta_{L S_{i}}$ is the corresponding least squares parameter estimate.

\section{III.5. Model performance estimation}

The performance index of the model is defined as its mean error over all possible adhesives in the family of interest, i.e. as the root of the expectation of its mean square error $E((y-$ $\left.\left.f\left(\xi, \boldsymbol{\theta}_{L S}\right)\right)^{2}\right)$.

Since the data set is small, we provide a leave-one-out (LOO) cross validation estimate of this performance index. The LOO estimate for a model obtained with $N$ adhesives is defined as follows: the $k$-th LOO error is the error for the $\mathrm{k}$-th adhesive made by the model obtained when the $k$-th adhesive is left out for the model parameter estimation, and the LOO estimate is the root of the mean of the $N$ squared LOO errors. These LOO errors can be computed conveniently as functions of the residuals of the model obtained on the whole data set, see Appendix 3. The root mean square error (RMSE) obtained on the whole data set is denoted by $\mathrm{RMSE}_{\mathrm{whole}}$, and that of LOO by RMSE $\mathrm{LOO}_{\text {. }}$

We also provide the RMSE obtained when estimating the parameters of the model on a training set of 20 adhesives for tack and 18 adhesives for peel, and testing the model on an independent 
test set of 6 adhesives corresponding to a series of different formulations (see Appendix 2). The RMSE obtained on the training set is denoted by $\mathrm{RMSE}_{\text {train }}$, and that on the test set by $\mathrm{RMSE}_{\text {test }}$. A good model is such that RMSE $\mathrm{LOO}_{\mathrm{RMSE}} \mathrm{Rhole}_{\text {wh }}$ and $\mathrm{RSE}_{\text {test }} / \mathrm{RMSE}_{\text {train }}$ are not much larger than 1 .

\section{Application to the prediction of the loop tack and peel values}

\section{IV.1. Using the lack of fit test}

For loop tack, with a degree 1, 15 monomials are needed for the lack of fit (LOF) test, but the RMSE $_{\mathrm{LOO}}$ and the RMSE $\mathrm{E}_{\text {test }}$ are large. With a degree 2, only 11 monomials are needed, and all the RMSEs are acceptable. For peel, with a degree 1, 16 monomials are needed for the LOF test, but the RMSE $E_{\mathrm{LOO}}$ and the RMSE $\mathrm{E}_{\text {test }}$ are also large. With a degree 2, only 9 monomials are needed, and all the RMSEs are acceptable. The performance of the tack and peel models of degree 2 is summarized in Table 1. We note that the models obtained with the LOF test possess either a RMSE $E_{L O O}$ or a RMSE $E_{\text {test }}$ that is large compared to the $\mathrm{RMSE}_{\text {whole }}$ or to the $\mathrm{RMSE}_{\text {train }}$.

\begin{tabular}{|c||c|c|c||c|c||c|c|}
\hline $\begin{array}{c}\text { output } \\
\text { property }\end{array}$ & $\begin{array}{c}\mathrm{nb} \\
\text { monomials } \\
\text { /descriptors }\end{array}$ & $\begin{array}{c}\text { nb PSAs } \\
\text { training/test }\end{array}$ & $\begin{array}{c}\text { output s.e. } \\
\text { estimate } v \\
(\mathrm{~N} / 25 \mathrm{~mm})\end{array}$ & $\begin{array}{c}\mathrm{RMSE}_{\text {whole }} \\
(\mathrm{N} / 25 \mathrm{~mm})\end{array}$ & $\begin{array}{c}\mathrm{RMSE}_{\mathrm{LOO}} \\
(\mathrm{N} / 25 \mathrm{~mm})\end{array}$ & $\begin{array}{c}\mathrm{RMSE}_{\text {train }} \\
(\mathrm{N} / 25 \mathrm{~mm})\end{array}$ & $\begin{array}{c}\mathrm{RMSE}_{\text {test }} \\
(\mathrm{N} / 25 \mathrm{~mm})\end{array}$ \\
\hline loop tack & $11 / 16$ & $20 / 6$ & 0.59 & 0.39 & 1.44 & 0.40 & 0.54 \\
\hline peel & $9 / 14$ & $18 / 6$ & 0.61 & 0.64 & 1.17 & 0.49 & 1.44 \\
\hline
\end{tabular}

Table 1. Model of degree 2 selected with the LOF test (100 descriptors).

The model sensitivities are shown in Figure 5. The 11 monomials of the tack model are, in the order of relevance: $\xi_{2} \xi_{8}, \xi_{6} \xi_{100}, \xi_{6} \xi_{70}, \xi_{40} \xi_{85}, \xi_{2} \xi_{31}, \xi_{59} \xi_{65}, \xi_{39} \xi_{84}, \xi_{41} \xi_{70}, \xi_{6} \xi_{8}, \xi_{44}$ $\xi_{57}, \xi_{25}{ }^{2}$. The 9 monomials of the peel model are: $\xi_{59}{ }^{2}, \xi_{5} \xi_{8}, \xi_{6} \xi_{75}, \xi_{59}, \xi_{23} \xi_{74}, \xi_{75} \xi_{78}, \xi_{9}$ $\xi_{24}, \xi_{44} \xi_{45}, \xi_{30} \xi_{57}$. Most of the monomials are hence cross-product monomials. This illustrates the benefits of the statistical approach, whose only assumption is that the information is contained in the raw descriptors: it discovers interaction effects between different parts of the curve which were not predictable $a$ priori, and which are still difficult to analyze.

Note also that the tack model involves descriptors located at the end of the curves, i.e. descriptors whose variability is generally high, whereas the peel model does not. Though it may seem surprising, the fact that relevant descriptors are located after the contact of certain tests is over is not pathological. It simply means that these descriptors will not contribute to the output of the model for these tests, whereas for other tests, they will contribute (positively or negatively).

The number of parameters of the models selected with the LOF test are too large given the training set size ( $N=26$ or 24 ), and the discrepancy between the RMSE $E_{\text {whole }}$ and the RMSE $E_{\mathrm{LOO}}$ are too large (a factor of 4 for tack, of 2 for peel). As a matter of fact, when increasing the 
number of descriptors, the correlation will always be better on the training set, but not necessarily on unseen PSAs. Furthermore, we do not have total confidence in the output variance estimate used for the LOF test, since the detail of the measurements is unknown for 16 adhesives out of 26 .
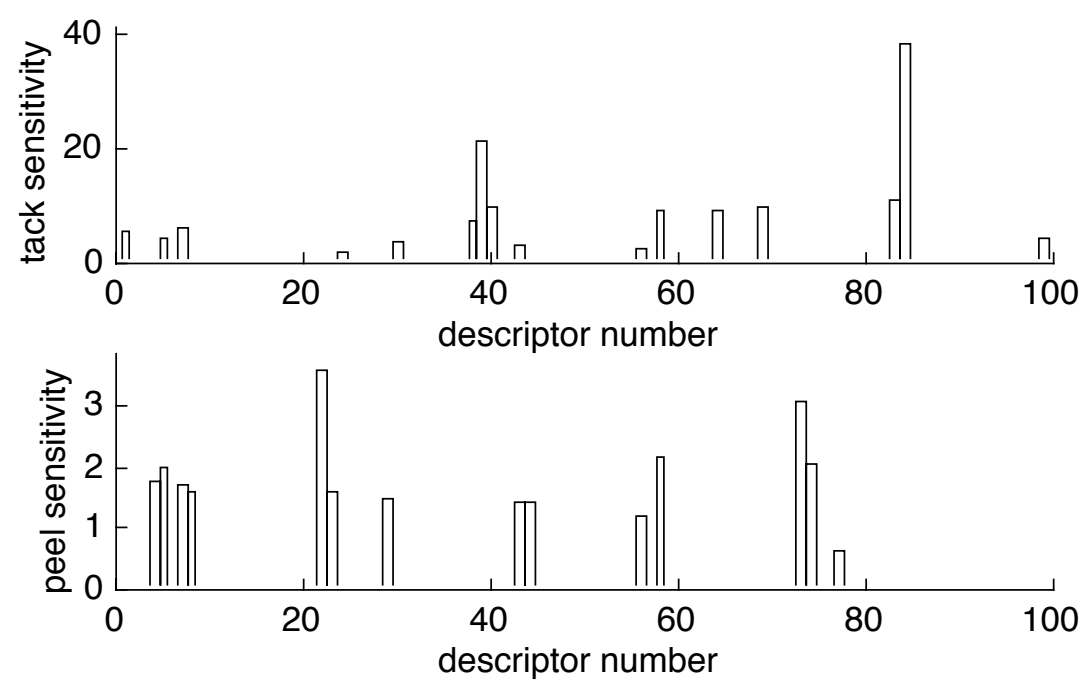

Figure 5. Sensitivities of the models selected with the LOF test (100 descriptors).

\section{IV.2. Achieving a bias/variance compromise}

Thus, we retained models with less monomials than those selected with the LOF test, but for which the discrepancy between the RMSE $E_{\text {whole }}$ and the $\mathrm{RMSE}_{\mathrm{LOO}}$ is smaller. In other words, we pay for reducing the model variability (variance) by accepting a lack of fit (bias). For tack, we retain a model with 9 monomials, and for peel, a model with 7 monomials, see Table 2 . The discrepancy between the $\mathrm{RMSE}_{\mathrm{whole}}$ and the $\mathrm{RMSE}_{\mathrm{LOO}}$ is smaller, and the number of parameters is more reasonable. However, the $\mathrm{RMSE}_{\mathrm{LOO}}$ and the $\mathrm{RMSE}_{\text {test }}$ of the tack model are still large as compared to the output standard error estimate $v$.

\begin{tabular}{|c||c|c|c||c|c||c|c|}
\hline $\begin{array}{c}\text { output } \\
\text { property }\end{array}$ & $\begin{array}{c}\mathrm{nb} \\
\text { monomials } \\
\text { /descriptors }\end{array}$ & $\begin{array}{c}\text { nb PSAs } \\
\text { training/test }\end{array}$ & $\begin{array}{c}\text { output s.e. } \\
\text { estimate } v \\
(\mathrm{~N} / 25 \mathrm{~mm})\end{array}$ & $\begin{array}{c}\mathrm{RMSE}_{\text {whole }} \\
(\mathrm{N} / 25 \mathrm{~mm})\end{array}$ & $\begin{array}{c}\mathrm{RMSE}_{\mathrm{LOO}} \\
(\mathrm{N} / 25 \mathrm{~mm})\end{array}$ & $\begin{array}{c}\mathrm{RMSE} \\
(\mathrm{N} / 25 \mathrm{~mm})\end{array}$ & $\begin{array}{c}\mathrm{RMSE}_{\text {trast }} \\
(\mathrm{N} / 25 \mathrm{~mm})\end{array}$ \\
\hline loop tack & $9 / 13$ & $20 / 6$ & 0.59 & 1.26 & 2.86 & 1.07 & 2.43 \\
\hline peel & $7 / 10$ & $18 / 6$ & 0.61 & 0.93 & 1.24 & 0.83 & 1.20 \\
\hline
\end{tabular}

Table 2. Model of degree 2 achieving the best bias/variance compromise (100 descriptors).

The sensitivities are shown in Figure 6. The 9 monomials of the tack model involve 13 descriptors, and the 7 monomials of the peel model involve 10 descriptors. The tack model again needs descriptors located at the end of the curves. 

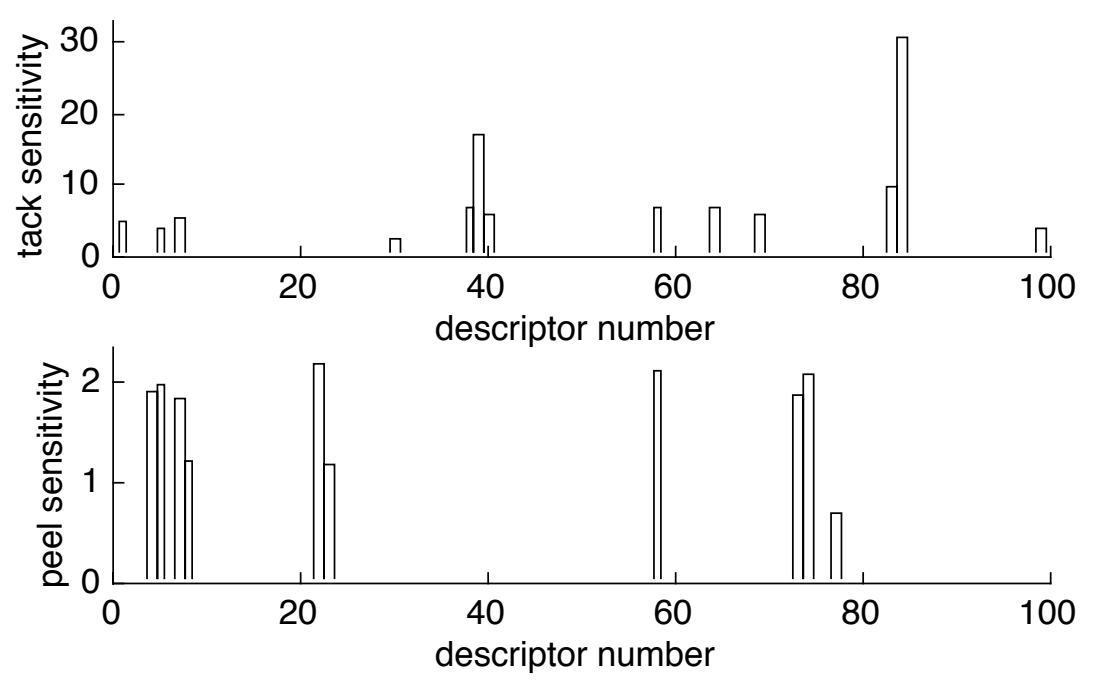

Figure 6. Sensitivities of the models achieving a bias/variance compromise (100 descriptors).

\section{IV.3. Achieving a bias/variance compromise and using only 60 descriptors}

The results are quite stable when the number of descriptors is in the interval $[50 ; 100]$. We present the results obtained with 60 descriptors, which are summarized in Table 3.

\begin{tabular}{|c||c|c|c||c|c||c|c|}
\hline $\begin{array}{c}\text { output } \\
\text { property }\end{array}$ & $\begin{array}{c}\mathrm{nb} \\
\text { monomials } \\
\text { /descriptors }\end{array}$ & $\begin{array}{c}\text { nb PSAs } \\
\text { training/test }\end{array}$ & $\begin{array}{c}\text { output s.e. } \\
\text { estimate } v \\
(\mathrm{~N} / 25 \mathrm{~mm})\end{array}$ & $\begin{array}{c}\mathrm{RMSE}_{\text {whole }} \\
(\mathrm{N} / 25 \mathrm{~mm})\end{array}$ & $\begin{array}{c}\mathrm{RMSE}_{\mathrm{LOO}} \\
(\mathrm{N} / 25 \mathrm{~mm})\end{array}$ & $\begin{array}{c}\mathrm{RMSE} \\
(\mathrm{N} / 25 \mathrm{~mm})\end{array}$ & $\begin{array}{c}\mathrm{RMSE}_{\text {train }} \\
(\mathrm{N} / 25 \mathrm{~mm})\end{array}$ \\
\hline loop tack & $9 / 14$ & $20 / 6$ & 0.59 & 1.38 & 2.19 & 1.30 & 2.00 \\
\hline peel & $7 / 11$ & $18 / 6$ & 0.61 & 0.88 & 1.14 & 0.79 & 1.28 \\
\hline
\end{tabular}

Table 3. Model of degree 2 achieving the best bias/variance compromise (60 descriptors).

The 9 monomials of the tack model are, in the order of relevance: $\xi_{2} \xi_{54}, \xi_{4} \xi_{39}, \xi_{3} \xi_{4}, \xi_{27}$, $\xi_{33} \xi_{49}, \xi_{1} \xi_{58}, \xi_{4} \xi_{33}, \xi_{22} \xi_{46}, \xi_{16} \xi_{35}$. The 7 monomials of the peel model are: $\xi_{41} \xi_{43}, \xi_{3} \xi_{5}$, $\xi_{38} \xi_{43}, \xi_{3} \xi_{34}, \xi_{45^{2}}, \xi_{28} \xi_{48}, \xi_{35} \xi_{42}$. Most of them are still cross-product monomials.
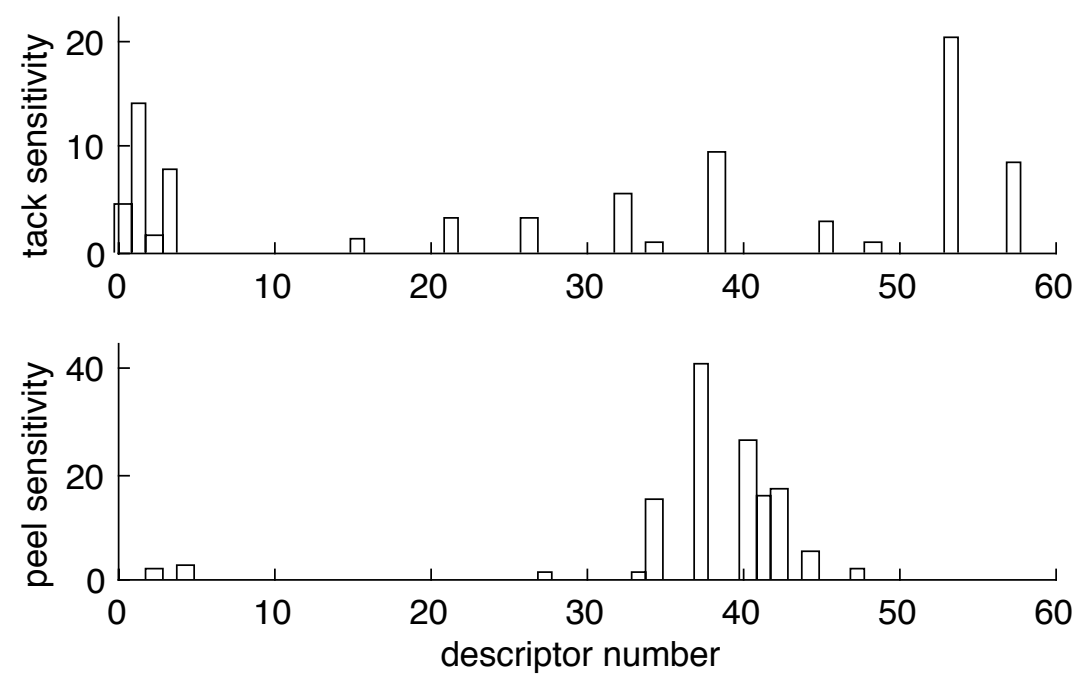

Figure 7. Sensitivities of the models achieving a bias/variance compromise (60 descriptors). 


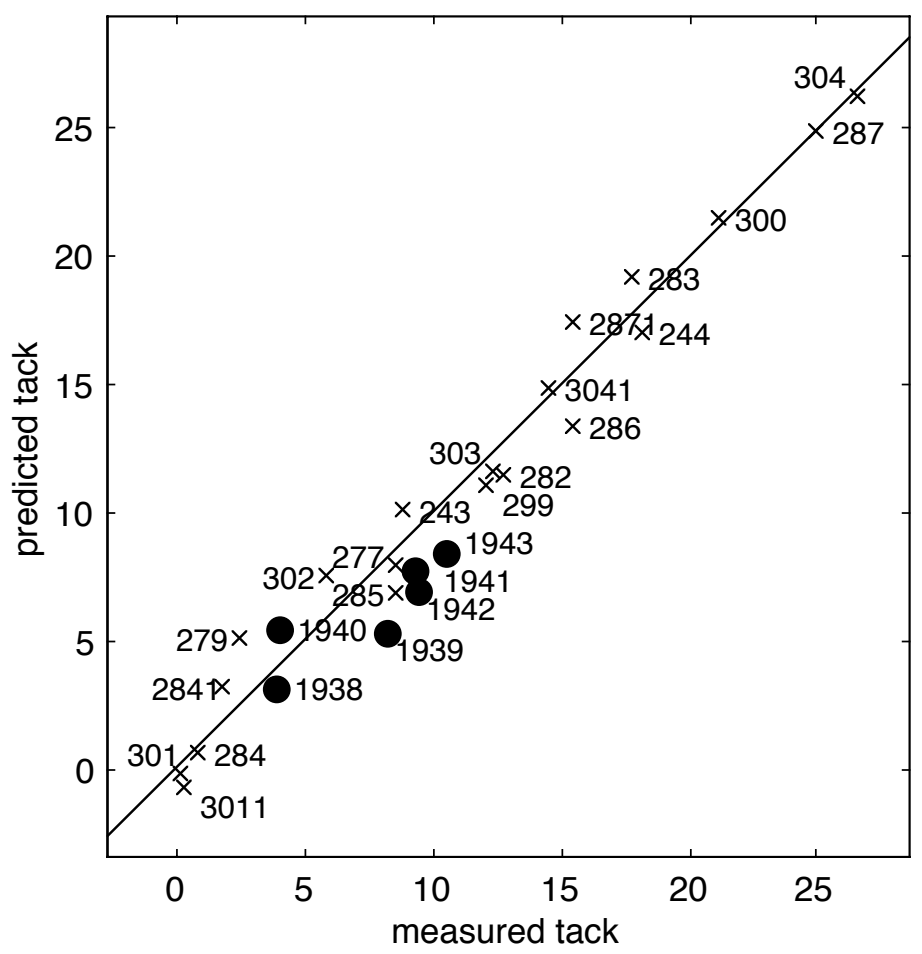

Figure 8. Predicted/measured tack, for 20 training (crosses) and 6 test adhesives (filled circles).

Figure 7 displays the model sensitivities. Figures 8 and 9 display the outputs of the models for the training and test adhesives. The LOO and test errors of the tack model are even smaller than with 100 descriptors, probably due to the averaging effect on the end of the curve.

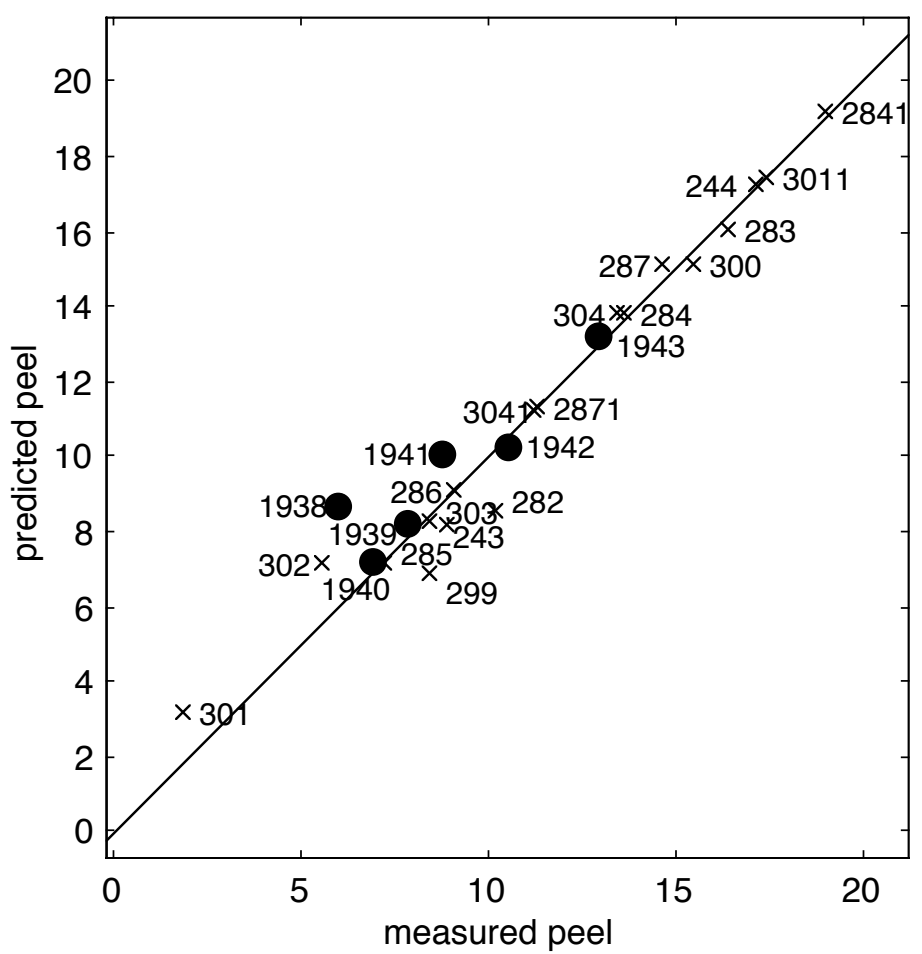

Figure 9. Predicted/measured peel, for 18 training (crosses) and 6 test adhesives (filled circles). 


\section{Discussion}

Let us first summarize the results. Table 3 shows that the peel model achieves RMSE $E_{L O O}$ and $\mathrm{RMSE}_{\text {test }}$ twice as large as the standard deviation, with a reasonably small number of parameters as compared to the available number of adhesives. The tack model is less accurate, with $\mathrm{RMSE}_{\mathrm{LOO}}$ and $\mathrm{RMSE}_{\text {test }}$ three times as large as the standard deviation, despite the larger number of parameters. The loop tack may be more difficult to predict for two reasons. First because the relationship between the descriptors and loop tack may be more nonlinear. Second because the tack model involves descriptors located at the end of the curves, where the probe test is less reproducible, whereas the peel model does not.

Aside from this assessment of the performance of the model from a purely statistical point of view, we can discuss in more details the advantages and limitations of the approach as presented here. A number of points need to be addressed:

- The adhesives for both the training set and the test set were all of the same family of adhesives (SIS + tackifying resins). It remains to be seen whether a model developed for a specific family of PSA would work to predict peel and loop test for a different family of PSA. Our guess is that as long as the interactions between the PSA and surface do not vary much, the model should be applicable to another family of adhesives such as acrylics. However more tests would be needed to confirm that.

- We did not discuss in this paper the influence of debonding mechanisms on the peel force values. It is well known that in a certain range of peel velocities, many PSA experience a socalled stick-slip debonding behaviour. In this case the peel force is no longer defined as a single value, but rather two values (an initiation force and an arrest force, which depend among other things on the details of the experimental setup). Clearly this type of mechanism cannot be predicted by our model, but one could envision that a different set of descriptors could be used to build a model able to predict the occurrence of stick slip.

- Although indeed the model does not use physically meaningful descriptors, it certainly assumes that the information necessary to predict the peel force or loop test is contained in the probe test curve. It uses in other terms the probe test curve as a signature of the material (like an infrared spectrum) and extracts the relevant bits of the signature to predict the peel force or loop test result. For this approach to be successful, it is important to minimize the variability of all the parameters that are not the properties of the adhesive itself. This means that all adhesive layers should have the same thickness and surface roughness, that the probe used for the probe tests should be the same for all tests, that the experimental conditions for the probe test (probe velocity, contact force and contact time) and for the loop test and peel test should be kept constant. 


\section{Conclusion}

The results we obtained confirm our starting hypothesis that the information about the loop tack and the peel is indeed contained in the force signal obtained with a probe test. Although the accuracy of the models is not yet optimal, we feel that the proposed procedure is very promising and is likely to provide increasingly more refined predictive models of standard PSA properties. This approach is complementary to an approach based on intuitively identifying the most relevant physically significant descriptors of the force-displacement curves such as the maximum stress or the adhesion energy. It is worthwhile to note that the most relevant descriptors found by the empirical model are often coupled and may provide ideas for more complex and better physically based descriptors.

As usual with empirical models, the quality of the model depends on the range and representativity of the data available to construct it, and we expect further improvements from a reliable estimation of the measurement variability for each adhesive, which will allow a correct weighting of the data points, and from the use of a larger, more representative data set. Such a model would have a great potential as a development and screening tool. Probe tests results, obtained easily and rapidly even from rather small amounts of materials, would then guide the synthetic chemist to select the most promising synthesis conditions, and to perform a timeconsuming complete set of tests on these selected formulations only.

We also feel that, because it probes the large strain nonlinear elastic properties of the materials, the probe test measurements may contain some of the information necessary to predict the resistance to shear. This is an industrially very important but notoriously difficult property to predict from the molecular structure of the adhesive, and we plan to apply the same statistical methodology in that direction.

\section{Acknowledgements}

We are very grateful to Hilde De Muyck for producing the results that motivated this study, as well as to Jan Vaneecke, who performed the major part of the experimental work. 


\section{Appendices}

\section{A1. Adhesive formulations}

\begin{tabular}{|c|c|}
\hline PSA N ${ }^{\circ}$ & Formulation \\
\hline 243 & D1161/Piccotac $212: 12 / 5$ \\
\hline 244 & D1161/Piccotac 212: $12 / 10$ \\
\hline 277 & D1161/Piccotac 212: $12 / 8$ \\
\hline 279 & D1161/Piccotac 212: $12 / 12$ \\
\hline 282 & D1161/Piccotac $95: 12 / 5$ \\
\hline 283 & D1161/Piccotac $95: 12 / 10$ \\
\hline 284 & D1161/Piccotac 95: $12 / 20$ \\
\hline 285 & D1161/Foral $85: 12 / 5$ \\
\hline 286 & D1161/Foral $85: 12 / 10$ \\
\hline 287 & D1161/Foral $85: 12 / 20$ \\
\hline 299 & D1160/Piccotac $95: 12 / 5$ \\
\hline 300 & D1160/Piccotac 95: $12 / 10$ \\
\hline 301 & D1160/Piccotac 95: $12 / 20$ \\
\hline 302 & D1160/Foral $85: 12 / 5$ \\
\hline 303 & D1160/Foral $85: 12 / 10$ \\
\hline 304 & D1160/Foral $85: 12 / 20$ \\
\hline 2841 & D1161/Piccotac 95E : $12 / 20$ \\
\hline 2871 & D1161/Foral 85: $12 / 20$ \\
\hline 3011 & D1160/Piccotac 95E : $12 / 20$ \\
\hline 3041 & D1160/Foral 85: $12 / 20$ \\
\hline 1938 & D1161/Foral $85: 12 / 8$ \\
\hline 1939 & D1161/Foral $85: 12 / 12$ \\
\hline 1940 & D1160/Foral $85: 12 / 8$ \\
\hline 1941 & D1160/Foral $85: 12 / 12$ \\
\hline 1942 & D1160/Piccotac $95: 12 / 8$ \\
\hline 1943 & D1160/Piccotac 95: $12 / 12$ \\
\hline
\end{tabular}

Table 4. Formulations of the 26 adhesives of the data set. 


\section{A2. Description of the data set}

\begin{tabular}{|c|c|c|c|c|c|}
\hline $\begin{array}{l}\text { PSA } \\
\mathrm{N}^{\circ}\end{array}$ & $\begin{array}{c}\text { number of } \\
\text { curves }\end{array}$ & $\begin{array}{c}M^{k}(\text { loop } \\
\text { tack) }\end{array}$ & $\begin{array}{c}\text { loop tack }(\mathrm{N} / 25 \\
\mathrm{mm}) \\
\text { mean } y^{k}\left(\operatorname{std} s^{k *}\right)\end{array}$ & $M^{k}($ peel $)$ & $\begin{array}{c}\operatorname{peel}(\mathrm{N} / 25 \mathrm{~mm}) \\
\text { mean } y^{k}\left(\operatorname{std} s^{k *}\right)\end{array}$ \\
\hline 243 & 5 & 3 & $8.8(-)$ & 3 & $8.9(-)$ \\
\hline 244 & 4 & 3 & $18.1(-)$ & 3 & $17.1(-)$ \\
\hline 277 & 3 & 3 & $8.6(-)$ & 0 & - \\
\hline 279 & 4 & 3 & $2.4(-)$ & 0 & - \\
\hline 282 & 5 & 3 & $12.8(-)$ & 3 & $10.2(-)$ \\
\hline 283 & 3 & 3 & $17.8(-)$ & 3 & $16.4(-)$ \\
\hline 284 & 4 & 3 & $0.8(-)$ & 3 & $13.6(-)$ \\
\hline 285 & 5 & 3 & $8.5(-)$ & 3 & $7.2(-)$ \\
\hline 286 & 5 & 3 & $15.5(-)$ & 3 & $9.1(-)$ \\
\hline 287 & 4 & 3 & $25(-)$ & 3 & $14.6(-)$ \\
\hline 299 & 3 & 3 & $12(-)$ & 3 & $8.4(-)$ \\
\hline 300 & 4 & 3 & $21.2(-)$ & 3 & $15.5(-)$ \\
\hline 301 & 4 & 3 & $0.2(-)$ & 3 & $1.8(-)$ \\
\hline 302 & 3 & 3 & $5.8(-)$ & 3 & $5.5(-)$ \\
\hline 303 & 3 & 3 & $12.4(-)$ & 3 & $8.4(-)$ \\
\hline 304 & 4 & 3 & $26.6(-)$ & 3 & $13.4(-)$ \\
\hline 2841 & 3 & 5 & $1.8(0.6)$ & 4 & $19(1.3)$ \\
\hline 2871 & 3 & 5 & $15.4(1.2)$ & 5 & $11.3(0.5)$ \\
\hline 3011 & 3 & 5 & $0.3(<0.1)$ & 5 & $17.4(3.3)$ \\
\hline 3041 & 3 & 5 & $14.5(2.5)$ & 5 & $11.2(1.1)$ \\
\hline 1938 & 5 & 4 & $3.9(<0.1)$ & 5 & $6.0(0.4)$ \\
\hline 1939 & 5 & 4 & $8.3(0.5)$ & 5 & $7.9(0.4)$ \\
\hline 1940 & 5 & 5 & $4.1(0.3)$ & 5 & $6.9(0.3)$ \\
\hline 1941 & 5 & 4 & $9.4(0.4)$ & 5 & $8.8(0.3)$ \\
\hline 1942 & 5 & 5 & $9.5(0.9)$ & 5 & $10.5(0.4)$ \\
\hline 1943 & 5 & 4 & $10.6(2.1)$ & 5 & $13.0(0.4)$ \\
\hline & & & $\mathrm{s}=1.19$ & & $\mathrm{~s}=1.23$ \\
\hline
\end{tabular}

Table 5. The available data set. (*) Standard deviations estimated with repeats, if available. The shaded cells correspond to the adhesives for which the standard deviations are available. 


\section{A3. Least squares and statistics}

We consider a data set of $N$ adhesives represented by the couples $\left\{\xi^{k}, y^{k}\right\}_{k=1}$ to $N$. The $\left\{\xi^{k}\right\}$ are the force values at $n$ sampling instants, and the $\left\{y^{k}\right\}$ are the corresponding mean values of the considered property, loop tack or peel. To assume that the information about the property is contained in the candidate descriptors $\{\xi\}$ is equivalent to assume the existence of the model:

$$
y=E(y)+w=g(\xi)+w
$$

where the mean $g$ is an unknown function of $\xi$, and $w$ is a zero mean random variable modeling uncertainty. We further assume homoscedasticity, i.e. that the outputs $\left\{y^{k}\right\}$ are uncorrelated and have the same variance $\lambda^{2}$.

\section{Polynomial model}

The goal is to choose a suitable parameterized function $f(\boldsymbol{\xi}, \boldsymbol{\theta})$ and a value $\boldsymbol{\theta}_{\text {opt }}$ of the parameters $\boldsymbol{\theta}$ such that $f\left(\boldsymbol{\xi}, \boldsymbol{\theta}_{\text {opt }}\right)$ is close to $g(\boldsymbol{\xi})$ in the domain of interest. Here, we consider a polynomial model with $p$ monomials and parameter $p$-vector $\boldsymbol{\theta}$. We denote the monomials of adhesive $k$ by $\boldsymbol{x}^{k}$, which is a $p$-vector, and whose first component is equal to one. For example:

$$
f\left(\xi^{k}, \boldsymbol{\theta}\right)=\theta_{1}+\theta_{2} \xi_{15}^{k}+\theta_{3}\left(\xi_{45}^{k}\right)^{2}+\theta_{4} \xi_{10}^{k} \xi_{31}^{k}=\theta_{1} x_{1}^{k}+\theta_{2} x_{2}^{k}+\theta_{3} x_{3}^{k}+\theta_{4} x_{4}^{k}=\left(x^{k}\right)^{T} \boldsymbol{\theta}
$$

The estimates of this model for the $N$ adhesives of the training set are hence given by:

$$
\left[\begin{array}{c}
\left(x^{1}\right)^{T} \\
\ldots \\
\left(x^{N}\right)^{T}
\end{array}\right] \boldsymbol{\theta} \equiv\left[\begin{array}{lllll}
1 & x_{2} & \ldots & x_{p}
\end{array}\right] \boldsymbol{\theta} \equiv X \boldsymbol{\theta}
$$

where the $\left\{\boldsymbol{x}_{i}\right\}$ are $N$-vectors. The $(N, p)$ matrix $X$ is known as the experiment matrix. Assuming that $N \geq p$ and $\operatorname{rank}(X)=p$, i.e. that the $\left\{\boldsymbol{x}_{i}\right\}$ are linearly independent, the least squares (LS) parameter estimate $\boldsymbol{\theta}_{L S}$ minimizing $\|\boldsymbol{y}-X \boldsymbol{\theta}\|^{2}$ is given by:

$$
\boldsymbol{\theta}_{L S}=\left(X^{T} X\right)^{-1} X^{T} \boldsymbol{y}
$$

The residual $N$-vector $\boldsymbol{r}$ is defined as the $N$-error vector of the LS model, that is:

$$
\boldsymbol{r}=\boldsymbol{y}-X \boldsymbol{\theta}_{L S}
$$

The residual sum of squares is equal to $\boldsymbol{r}^{T} \boldsymbol{r}$.

\section{Null hypothesis}

Let us consider the hypothesis that there exists an unknown parameter vector $\boldsymbol{\theta}^{*}$ such that:

$$
H_{0}: \boldsymbol{y}=E(\boldsymbol{y})+\boldsymbol{w}=X \boldsymbol{\theta}^{*}+\boldsymbol{w}
$$

and where hence $E(\boldsymbol{w})=\boldsymbol{0}$. This hypothesis is called the null hypothesis $\left(H_{0}\right)$ : it is the hypothesis that the monomials $\left\{x_{i}\right\}$ are sufficient to explain the output property $y$, i.e. that the model is unbiased. In other words, the subspace spanned by the $\left\{\boldsymbol{x}_{i}\right\}$ (the image of the matrix $X$ ) contains the mean of $\boldsymbol{y}$. If the monomials $\left\{x_{i}\right\}$ are not sufficient, $H_{0}$ is false, which means that the model is biased. Since we search for an unbiased model, $H_{0}$ is the hypothesis we want to test. 
If $H_{O}$ is true, $E\left(\boldsymbol{\theta}_{L S}\right)=\boldsymbol{\theta}^{*}$. Moreover, the noise being homoscedastic, the residuals provide an unbiased estimate of the variance $\lambda^{2}$ of the outputs $\left\{y^{k}\right\}$ :

$$
E\left(\frac{\boldsymbol{r}^{T} \boldsymbol{r}}{N-p}\right)=\lambda^{2}
$$

\section{Lack of fit test}

Suppose that we dispose of an independent estimate $v^{2}$ of the variance $\lambda^{2}$ that is reliable, i.e. unbiased: $E\left(v^{2}\right)=\lambda^{2}$. Intuitively, if $H_{0}$ is true, the residual vector $\boldsymbol{r}$ is essentially due to uncertainty and the following ratio $f$ should be close to 1 :

$$
f=\frac{\frac{\boldsymbol{r}^{T} \boldsymbol{r}}{N-p}}{v^{2}}
$$

On the other hand, if $H_{0}$ is false, the model is too simple (biased) and $\boldsymbol{r}$ is not only due to uncertainty but also to a model bias, and the ratio $f$ will be much larger than 1 .

If we assume Gaussian outputs, it is easy to test whether $f$ is significantly larger than 1 , see [Draper \& Smith 1998] [Rivals \& Personnaz 2003b]. Then, if $v^{2}$ is obtained from $\mathrm{q}$ independent values:

$$
\frac{q v^{2}}{\lambda^{2}} \rightarrow \chi^{2}(q)
$$

where $\chi^{2}(q)$ denotes the Pearson distribution with $q$ degrees of freedom. If moreover $H_{0}$ is true, we have:

$$
\frac{\boldsymbol{r}^{T} \boldsymbol{r}}{\lambda^{2}} \rightarrow \chi^{2}(N-p)
$$

and hence:

$$
f=\frac{\frac{\boldsymbol{r}^{T} \boldsymbol{r}}{N-p}}{v^{2}} \rightarrow \text { Fisher }(N-p, q)
$$

This leads to the lack of fit (LOF) test. Let us choose a value $\alpha$ for the probability of rejecting $H_{0}$ when it is true, the type I risk. Then, we will decide to reject $H_{0}$ when:

$$
f=\frac{\frac{\boldsymbol{r}^{T} \boldsymbol{r}}{N-p}}{v^{2}}>F_{N-p, q}(1-\alpha)
$$

where $F_{N-p, q}(1-\alpha)$ denotes the $1-\alpha$ quantile of the Fisher distibution with $N-p$ and $q$ degrees of freedom. In other words, if the value of $f$ is too large given $\alpha$, we will decide that the model is biased with a risk $\alpha$ of being wrong.

\section{Independent variance estimate}

The available individual measurements $\left\{y^{k, j}\right\}_{k=17 \text { to } 26}$ of the property are assumed to have a common variance $\sigma^{2}$. Hence, we have the following unbiased estimate $s^{2}$ of $\sigma^{2}$ :

$$
s^{2}=\frac{\sum_{k=17}^{26}\left(M^{k}-1\right)\left(s^{k}\right)^{2}}{M-10}=\frac{\sum_{k=17}^{26} \sum_{j=1}^{M^{k}}\left(y^{k, j}-y^{k}\right)^{2}}{M-10}
$$


where, for each adhesive $k, M^{k}$ is the number of repeats, $y^{k, j}$ is the value of the $j$-th repeat, $y^{k}$ is the mean value over the repeats, and $M=\sum_{k=17}^{26} M^{k}$.

Assuming Gaussian measurements:

$$
s^{2} \frac{M-10}{\sigma^{2}} \rightarrow \chi^{2}(M-N)
$$

The outputs being a mean of $M^{k}=3$ to 5 repeats, we choose to approximate $M^{k} \approx m=4$. Thus, we can consider $\lambda^{2}=\sigma^{2} / m$ as the common variance of the outputs $\left\{y^{k}\right\}$, and $v^{2}=s^{2} / m$ as its estimate, obtained with $M-10$ degrees of freedom.

\section{Leave-one-out errors}

The LS parameter vector $\boldsymbol{\theta}_{L S}$ of the model is obtained using the whole data set of size $N$. The residual vector of this model is $\boldsymbol{r}=\left[r^{1} \ldots r^{N}\right]^{T}=\boldsymbol{y}-X \boldsymbol{\theta}_{L S}$. The $k$-th leave-one-out (LOO) error is the error made on adhesive $k$ by the model obtained when adhesive $k$ is left out from the data set. The latter does not need to be computed, since the $k$-th LOO error is a simple function of the $k$-th residual $r^{k}$ :

$$
e_{L O O}^{k}=\frac{r^{k}}{1-h^{k}}
$$

where $h^{k}$ is the $k$-th diagonal term of the $(N, N)$ orthogonal projection matrix $X\left(X^{T} X\right)^{-1} X^{T}$ on the image of $X$, or "hat" matrix, see [Rivals \& Personnaz 2003a]. 


\section{A2. Alternative choice of physical descriptors}

We constructed 18 descriptors of the shape of the force signal as a function of time. These new inputs are the following :

1) The area below zero.

2) The area above zero.

3) The initial slope of the curve.

4) The slope of a line joining the point at which the force reaches $-1 \mathrm{~N}$ and the point at which the force reaches its first maximum.

5) The slope of a line joining the point at which the force reaches its first maximum and the point at which the force reaches the next minimum.

6) The slope of a line joining the point at which the force reaches this minimum and the point at which the force reaches the second maximum.

7) The slope of a line joining the point at which the force reaches its second maximum and the point at which the force reaches zero.

8) The instant when the force reaches $-1 \mathrm{~N}$.

9) The instant when the force crosses zero.

10) The instant when the force reaches its first maximum.

11) The instant when the force reaches the next minimum.

12) The instant when the force reaches its second maximum.

13) The instant when the force reaches zero.

14) The value of the first maximum of the force.

15) The value of the next minimum of the force.

16) The value of the second maximum of the force.

17) The number of force maxima.

18) The curvature at the minimum.

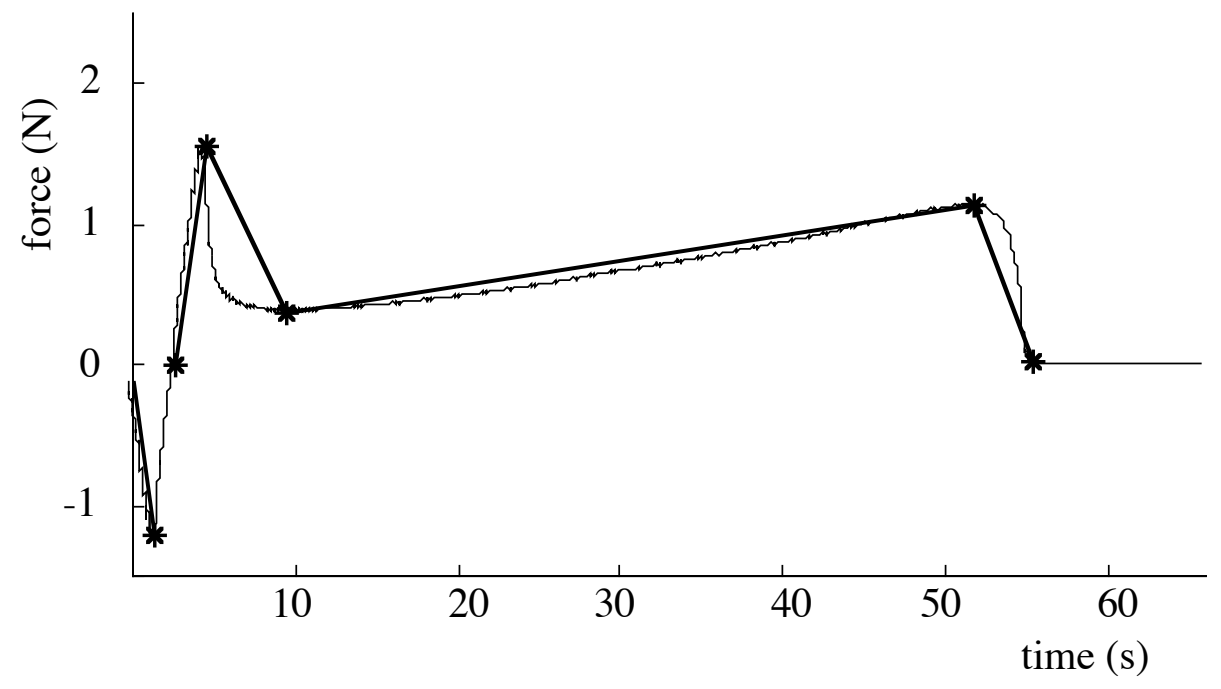

Figure 10. Some of the descriptors. 


\section{References}

K. Brown, J. C. Hooker, and C. Creton, Macromolecular Materials and Engineering 287, 163-179 (2002).

H. K. Chuang, C. Chiu, and R. Paniagua, Adhesives Age, 18-23 (1997).

C. Creton, J. C. Hooker, and K. R. Shull, Langmuir 17, 4948-4954 (2001).

C. Creton, MRS Bulletin 28, 434-439 (2003).

A. J. Crosby and K. R. Shull, Journal of Polymer Science: Part B: Polymer Physics 37, 34553472 (1999).

N. R. Draper and H. Smith. Applied regression analysis. John Wiley \& Sons inc., New York (1998).

G. H. Golub and C.F. Van Loan. Matrix computations. John Hopkins University Press, Baltimore, third edition (1983).

J. C. Grunlan, D. L. Holguin, H. K. Chuang, I. Perez, A. Chavira, R. Quilatan, J. Akhave, and A. R. Mehrabi, Macromolecular Rapid Communications 25, 286-291 (2004).

V. Kulikov, V. M. Mirsky, T. L. Delaney, D. Donoval, A. W. Koch and O. S. Wolfbeis, Measurement Science and Technology 16, 95-99 (2005).

H. Lakrout, P. Sergot, and C. Creton, Journal of Adhesion 69, 307-359 (1999).

H. Lakrout, C. Creton, D. Ahn, and K. R. Shull, Macromolecules 34, 7448-7458 (2001).

PSTC, Test methods for pressure sensitive adhesive tapes, 13th ed. (Pressure Sensitive Tape Council, 2000).

I. Rivals and L. Personnaz, Journal of Machine Learning Research 3, 1383-1398 (2003a).

I. Rivals and L. Personnaz, IEEE Transactions on Neural Networks 14, 804-819 (2003b).

A. Roos and C. Creton, Macromolecular Symposia 214, 147-156 (2004).

K. R. Shull and C. Creton, Journal of Polymer Science: Part B: Polymer Physics 42, 40234043 (2004).

H. Zhang, R. Hoogenboom, M. A. R. Meier and U. S. Schubert, Measurement Science and Technology 16, 203-211 (2005).

A. Zosel, Colloid and Polymer Science 263, 541-553 (1985). 